\title{
An Optimum Adaptive Parameterized Mask NHA Based Image Denoising
}

\author{
K.INDUPRIYA ${ }^{* 1}$, Dr. G. P. RAMESH KUMAR ${ }^{2}$ \\ ${ }^{* 1}$ Research Scholar, Department of Computer Science, SNR Sons College, Tamilnadu, India, \\ *11 indupriya1406@gmail.com \\ ${ }^{2}$ Head and Department of Computer Science, Government Arts college, Kulithalai, Tamilnadu, India, \\ 2'gpr.snr@gmail.com
}

\begin{abstract}
In image processing, the most significant challenges have been addressed due to the image denoising. Since, the segmentation of the original image components from the noisy image has high complexity. Therefore, the different image segmentation techniques were developed for effective segmentation by categorizing the noisy images. Among different methods, the image denoising including with the edge-preserving and segmentation according to the Mask Non-Harmonic Analysis (NHA) was the most utilized. In which, the image denoising was achieved in spatial domain by edge-preserving with fuzzy boundaries. In addition, the edge segmentation was performed by using the different segmentation parameters. However, the determination of parameter values was difficult for different noisy images. Moreover, the pre-determined threshold value was applied for the edge detection. Thresholding approach was applied for suppressing the unwanted information. However, the threshold value selection was required different values with respect to the different noise intensity images. Hence, in this paper, the machine learning algorithm Support Vector Machine (SVM) is proposed for determining the segmentation parameters automatically. The proposed algorithm is utilized for learning the segmentation parameters and output accuracy according to the noise levels and image frequency ranges. In addition, the edge detection is achieved by selecting the different threshold values based on the firefly optimization algorithm. Finally, the experimental results prove that the proposed Optimum Adaptive parameterized Mask NHA (OAMNHA) based image denoising has better performance than the other state-of-arts techniques.
\end{abstract}

Keywords-Image denoising, Image segmentation, Edge-preserving, Mask NHA, Edge detection, SVM, Firefly algorithm

\section{INTRODUCTION}

Image denoising is also known as noise reduction and is defined as the process of removing the noise from an image or a signal. In image processing, the most important process is the image denoising and also it act as the component for other processes. The major objective of image denoising process is removing the noise while preserving the edges [1]. Nowadays, digital images play the most vital role in different applications like satellite television, magnetic resonance imaging, computer tomography and geographical information systems. Generally, the images captured by the sensors are stinking by the noise [2]. Therefore, the data of interest is degraded due to the imperfect devices, data acquisition process issues and natural phenomena interference. In addition, the noise is introduced by the transmission errors and compression. Hence, the image denoising process is required as initial process in the image analysis for compensating the data corruption [3].

But, still image denoising process has different challenges since the artifacts are introduced by removing the noise which causes the blurring of images. Hosotani, F., et al. [4] proposed the Image Denoising process with the Edge-Preserving and Segmentation based on the Mask NHA (M-NHA). Here, the zero-mean white Gaussian noise is removed by using the high-resolution frequency analysis. The regions with the homogeneous texture in the noisy image are analyzed. The non-uniform regions which are acquired by segmentation are analyzed by using the Mask NHA for improving the Peak Signal-To-Noise Ratio (PSNR). However, the segmentation parameters optimization is not effectively achieved. Moreover, the threshold value for edge detection is predetermined which is determined for suppressing the unwanted information. This is not efficient for different images with different noise intensity values.

Hence, in this paper, an efficient image denoising technique is proposed based on the optimization algorithms. The proposed image denoising technique consists of two optimization algorithms such as machine learning SVM and firefly algorithm. The machine learning SVM algorithm is utilized for learning the segmentation parameters such as band parameter, filter intensity and edge sensibility. The firefly algorithm is proposed for optimizing the threshold values for different noisy images. Thus, the proposed image denoising approach is useful for removing the noise from noisy images by using the edge detection and edge segmentation process automatically. 
The remaining section of the article is scheduled as follows: Section II investigates about the different image denoising approaches which are related to the proposed system. Section III explains the methodology of proposed image denoising approach. Section IV illustrates the experimental results by using the simulation images. Section V concludes and discusses about the proposed work.

\section{LITERATURE SURVEY}

Yoshizawa, T., et al. [5] proposed high-resolution frequency analysis for noise reduction in periodic signals. The proposed frequency analysis was introduced for avoiding the issues in detecting the frequency variations rapidly since, the time resolution was low. In the proposed system, Non-Harmonic Analysis (NHA) was applied which consists of high frequency resolution including with the limited influence of frame length to the noise reduction issue. The utilization of NHA was investigated as an alternative pre-processing technique to DFT for noise reduction. However, the computation time complexity was high.

Xiao, Y., et al. [6] investigated about the restoration of images which are corrupted by Gaussian plus image noise through $l_{1}-l_{0}$ minimization approach. In the proposed minimization approach, $l_{1}$ was used for impulse denoising and $l_{0}$ was used for sparse representation over particular unknown dictionary of image patches. The proposed algorithm has three stages. The primary stage was used for identifying the outlier candidates. The second stage was used for recovering the image through the dictionary on the free-outlier pixels. At last, an alternating minimization algorithm was deployed for resolving the proposed minimization energy function. However, the outlier detection accuracy was required further improvement by utilizing the various noise detectors.

Hasegawa, M., et al. [7] proposed the novel technique for avoiding the constraints in image restoration by predicting the signal according to the NHA technique. The proposed NHA was used for extracting the precise spectra, irrespective of the window function and its frequency resolution which is less than that of DFT. The new textures were generated sequentially based on the spectrum acquired by NHA. The missing regions from the spectrum were repaired by using an improved cost function for 2D NHA. However, the computational complexity was high.

Yang, S., et al. [8] proposed image noise reduction method. In the proposed system, the structural similarities and sparse prior of image patches were exploited. In addition, the novel dictionary learning and similarity regularization based image noise reduction approach were proposed. The image noise reduction was formulated as the multiple variables optimization problem and the variables were alternatively optimized for acquiring the denoised image. However, the complexity of the proposed approach was high.

Zhang, J., \& Hirakawa, K. [9] proposed an improved denoising method through the Poisson mixture modeling of image sensor noise. The objective of the proposed method was to compare the real image sensor noise distribution to the models of noise which are considered in image denoising designs. In the proposed system, the novel Poisson mixture noise model was introduced for correcting the mismatch of tail behaviour. According to the noise model mismatch results, the mixture of Poisson denoising method was proposed for removing the denoising artifacts without affecting the information of images like edges and textures. However, the complexity of the proposed approach was approximately twice that of the conventional Poisson image denoising methods.

Xiong, R., et al. [10] proposed the novel image denoising algorithm according to the adaptive signal modeling and regularization. In the proposed approach, the non-stationary of image signals and the diversity among different transform models were addressed by employing the content-dependent adaptive models. The distribution model was estimated adaptively for each patch individually. Moreover, the expectation and variance parameters for each band of the certain patch were estimated by using the non-local correlation in image which is used for collecting the group of highly similar patches. The image was restored through the bandwise adaptive soft-thresholding and according to the Laplacian approximation of the distribution of similar-patch group transform coefficients. However, the actual coefficient distribution was not generalized Gaussian effectively.

Ma, H., \& Nie, Y. [11] proposed an enhanced anisotropic diffusion models for image denoising. The proposed improved model was utilized for classifying the different information of images like smooth regions, edges, corners and isolated noises by using the characteristic parameters and gradient variance parameter. Furthermore, an edge fusion method was proposed for edge preserving after denoising by fusing the various denoising and edge detection methods. Initially, the denoised images were acquired by using denoising methods and then the edge images of denoised images were obtained by edge detection methods. After that, the selected edge images were fused with more edges for replacing the edges of denoised images.

Fan, F., et al. [12] proposed hyperspectral image denoising along with the superpixel segmentation and lowrank representation. Initially, the Superpixel Segmentation (SS) was integrated into the Low-Rank Representation (LRR) for introducing the proposed novel denoising method named as SS-LRR. The Principal Component Analysis (PCA) was adopted for acquiring the primary principal component of Hyper Spectral 
Image (HIS). Then, the SS was adapted to the primary principal component of HIS for obtaining the homogeneous regions. Finally, the LRR approach was employed to each homogeneous region of HSI.

\section{PROPOSED METHODOLOGY}

In this section, the proposed image denoising methods are discussed briefly. The image analysis using DFT and DCT are providing the sidelobes while the signal period in the analysis window is not an integer value. Therefore, the sidelobes and noise are not completely differentiated from the amplitude of DFT and DCT. In addition, the sidelobe reduction is achieved in thresholding denoising. Hence, the method is required for solving the analysis of sidelobe suppression problem. Therefore, NHA is mostly suited for thresholding. In the proposed system, Optimum Adaptive Mask NHA (OAM-NHA) is proposed for removing the artifacts of denoised images. The proposed algorithm is performed as follows:

\section{A. $2 D$ NHA}

Generally, Mask NHA is defined by using 2D NHA in which the 2D DFT is represented in the spatial domain as,

$$
X(k, l)=\sum_{k=0}^{N-1} \sum_{l=0}^{M-1} I\left(n_{1}-n_{2}\right) e^{-j 2 \pi\left(\frac{k n_{1}}{N}+\frac{l n_{2}}{M}\right)}
$$

In equation (1), $I$ refers the image signal, $N$ and $M$ are the data sizes. The completely periodic signal in an $N \times M$ analysis window is assumed by the short Fourier transform. The frequency resolution of DFT is limited by the integer period of $\frac{k}{N}$. If the signal period in the analysis window is non-integer value then the sidelobes are observed. The Fourier coefficients are estimated by the sinusoidal wave fitting [7] and the 2D sinusoidal model is expressed as follows:

$$
I\left(n_{1}, n_{2}\right)=\hat{A} \cos \left(2 \pi \frac{\hat{f}_{x}}{f_{x_{s}}} n_{1}+\frac{\hat{f}_{y}}{f_{y_{s}}} n_{2}+\widehat{\varnothing}\right)
$$

In equation (2), $\hat{A}$ refers the amplitude of the sinusoidal model, $\hat{f}_{x}$ and $\hat{f}_{y}$ are the spatial frequencies, and $\widehat{\varnothing}$ is the phase parameter of the model. The sampling frequencies are denoted as $f_{x_{s}}$ and $f_{y_{s}}$ and are defined as $f_{x_{S}}=\frac{1}{\delta x}$ and $f_{y_{s}}=\frac{1}{\delta y}$ respectively where $x$ and $y$ are referred as two axes of the image domain. The 2D NHA is used for estimating the frequency by reducing the Mean Square Error (MSE) between the target signal and the model signal and is computed by the steepest descent method. The frequency parameters are computed as,

$$
F\left(\hat{A}, \hat{f}_{x}, \hat{f}_{y}, \widehat{\varnothing}\right)=\frac{1}{N_{1} N_{2}} \sum_{n_{1}=0}^{N_{1}-1} \sum_{n_{2}=0}^{N_{2}-1}\left\{I\left(n_{1}, n_{2}\right)-\hat{I}\left(n_{1}, n_{2}\right)\right\}^{2}
$$

The equation (3) is solved as an optimization problem by using the 2D DCT coefficients as the primary value. Generally, the frequency resolution is depending on the window size in the frequency analysis using DCT method. The original signal is distorted by removing the sidelobe via thresholding. Therefore, the sidelobes are suppressed by NHA which utilizes the high frequency resolution.

\section{B. Mask NHA}

The image denoising using domain transformation is employed by the block unit named as patch. The smoothing effectiveness of denoising is increased with the size of patch. The edge is converted into the sinc function by using DFT. While the part of sinc function in the frequency domain is removed by using thresholding, then the restored image in consisting of ringing artifacts. The edge information is vanished by thresholding since the sidelobes are occurred as the result of the edge analysis. The input signal in the analysis window is required for masking in order to minimize the influence of signal non-stationarity. Therefore, the masking region is acquired from the image segmentation results.

The noisy image is segmented as edge region and texture region during segmentation process. In addition, the texture region is segmented as multiple regions by using the spatial similarity. Hence, the target region is denoted as $\Omega$, and the remaining region is denoted as $\bar{\Omega}$. The weighting factor $w\left(n_{1}, n_{2}\right)$ is generated by using the binary information for differentiating $\Omega$ and $\bar{\Omega}$. The generated weighting factor is called as the masking matrix. The weighting factor is 0 while the outer region is represented in an image $I$ and the weighting factor is 1 when the target region is represented in an image $I$. The cost function is improved as follows,

$$
F\left(\hat{A}, \hat{f}_{x}, \hat{f}_{y}, \widehat{\varnothing}\right)=\frac{1}{N_{1} N_{2}} \sum_{n_{1}=0}^{N_{1}-1} \sum_{n_{2}=0}^{N_{2}-1} w\left(n_{1}, n_{2}\right) \times\left\{I\left(n_{1}, n_{2}\right)-\hat{I}\left(n_{1}, n_{2}\right)\right\}^{2}
$$

The cost function is used for extracting the spectrum from $\Omega$ excluding $\bar{\Omega}$. While the primary values are assigned by the Fast Fourier Transform (FFT), $\Omega$ must be given the temporary value.

\section{Edge-Preserving Segmentation}

The images are segmented into different clusters without edges for preventing the ringing artifacts. The segmentation of noisy images is containing the fuzziness in the choice of the region boundary since the determination of the boundary of a region within the noisy image is complex and provides the errors. Hence, the 
edge regions are defined by using the fuzzy boundaries. Therefore, initially the edges are extracted from the noisy images and then the edge regions are defined. The texture boundaries are used for reducing the boundary distortion during segmentation.

Hence, the edges are detected by using the canny edge detection approach. Before edge detection, the smoothing process is utilized by the canny edge detection method. For smoothing process, bilateral filtering is used by the detection approach [13]. The bilateral filtering is defined as the edge-preserving filter which utilizes the weights based on the difference of pixel luminance between the pixel of interest and its neighboring pixels. The bilateral filtering is denoted as,

$$
\begin{gathered}
F(x)=\frac{\int_{-\infty}^{\infty} \int_{-\infty}^{\infty} I(\xi) w(\xi, x) d \xi}{\int_{-\infty}^{\infty} w(\xi, x) d \xi} \\
w(\xi, x)=\exp \left(\frac{-(\xi-x)^{2}}{2 \sigma_{d}^{2}}\right) \exp \left(\frac{-(I(\xi)-I(x))^{2}}{2 \sigma_{r}^{2}}\right)
\end{gathered}
$$

In equation (5), $I$ and $F$ are referred as the input image and the output image correspondingly. Also, $x$ and $\xi$ are spatial variables and $\sigma_{d}$ and $\sigma_{r}$ are defined by the standard deviation, characterized the filter intensity and edge sensibility. Once the edges are detected, then the edge segmentation is performed based on the region growing and region merging.

Region growing is defined as the technique for iteratively expanding the initial region and the region merging is the process of combining the over-segmented results. The mean shift-based image segmentation is utilized for searching the local maximum density points in the feature space [14]. The images are segmented by applying the mean shift to the image feature space. The mean shift is utilized in the kernel density function as follows,

$$
\hat{f}_{h, K(x)}=\frac{c_{k, d}}{n h^{d}} \sum_{n=1}^{n} k\left(\left\|\frac{x-x_{i}}{h}\right\|^{2}\right)
$$

In equation (7), $x$ refers the variable vector, $x_{i}$ are sample vectors, $k$ is the kernel function, $h$ is the band parameter, and $c_{k, d}$ represents the normalization constant. The curved surface is constructed by the kernel density function and its local maximum is derived by the iteration process. The derived function for $k(x)$ is expressed as,

$$
g(x)=-k^{\prime}(x)
$$

Hence, the recursion for convergence to the local maximum is represented as follows:

$$
y_{j+1}=\frac{\sum_{i=1}^{n} x_{i} g\left(\left\|\frac{y_{j}-x_{i}}{h}\right\|^{2}\right)}{g\left(\left\|\frac{y_{j}-x_{i}}{h}\right\|^{2}\right)}, j=1,2, \ldots
$$

Image segmentation using mean shift is achieved by grouping according to the distance between each convergence point $y_{i, 1}=x_{i}$ and its neighboring points.

\section{Machine Learning SVM Algorithm}

In the proposed algorithm, the image segmentation is completely depends on the segmentation parameters such as band parameter, filter intensity and edge sensibility. Therefore, the best optimum parameter values are chosen by using machine learning SVM algorithm which is utilized for detecting the outliers effectively [15]. The SVM is constructed as a hyperplane or group of hyperplanes in a high or infinite-dimensional space. The accurate segmentation is achieved by the hyperplane which has the largest distance to the closest training data point of any class called functional margin. The training database is denoted as $D=\left\{\left(x_{1}, y_{1}\right), \ldots,\left(x_{n}, y_{n}\right)\right\}$, where $x_{i} \in R^{n}, y_{i} \in\{-1,+1\}$ represents two different classes. The optimal hyperplane with the maximal margin is obtained by SVM for segmenting two classes which requires for solving the following optimization problem.

$$
\begin{aligned}
& \max \sum_{i=1}^{n} \alpha_{i}-\frac{1}{2} \sum_{i, j=1}^{n} \alpha_{i} \alpha_{j} y_{i} y_{j} K\left(x_{i}, x_{j}\right) \\
& \text { Subject to } \sum_{i=1}^{n} \alpha_{i} y_{i}=0
\end{aligned}
$$

Where $0 \leq \alpha_{i} \leq b, i=1,2, \ldots, n$ and $\alpha_{i}$ represents the weight of training sample $x_{i}$ which is called as support vectors while $\alpha_{i}=0$ and $b$ denotes the regulation parameter utilized for trade-off the training accuracy and the model complexity. The kernel function $K$ is used for similarity measure between two samples and is defined as Radial Basis Function (RBF).

$$
K\left(x_{i}, x_{j}\right)=\exp \left(-\gamma\left\|x_{i}-x_{j}\right\|^{2}\right), \gamma>0
$$


Once the weights are estimated, then the test sample $x$ is classified as,

$$
\begin{aligned}
& y=\operatorname{Sign}\left(\sum_{i=1}^{n} \alpha_{i} y_{i} K\left(x_{i}, x_{j}\right)\right) \\
& \operatorname{Sign}(i)=\left\{\begin{array}{c}
+1, \text { if } i>0 \\
-1, \text { Otherwise }
\end{array}\right.
\end{aligned}
$$

The cross validation process is used for determining the values of $\langle\gamma, b\rangle$ which is carried out on the training database. The cross validation is used for estimating the generalization capability on new samples which are not in the training dataset. The training database is segmented into $k$ equal-sized subsets by using the $k$-fold cross validation and classifier is constructed by using the remaining samples for classification. This process is continued for $k$ times on each subset for obtaining the cross validation over the entire training database. Based on the classification, the optimal segmentation parameter values are selected for image segmentation process effectively.

Algorithm: SVM based Parameter Optimization

1. Initialize the input sample $x$ to classify

2. Consider the training database $D=\left\{\left(x_{1}, y_{1}\right), \ldots,\left(x_{n}, y_{n}\right)\right\}$

3. Assume $k$ number of nearest neighbors

4. //Output

5. Decision $y_{p} \in\{-1,1\}$

6. Obtain $k$ sample $\left(x_{i}, y_{i}\right)$ with minimal values of $K\left(x_{i}, x_{j}\right)-2 * K\left(x_{i}, x\right)$

7. Train the SVM model on the selected $k$ samples

8. Classify $x$ and obtain the result $y_{p}$

9. Return $y_{p}$

\section{E. Firefly Algorithm based Thresholding}

Furthermore, the edge detection of denoised image is performed based on the threshold values. In the proposed system, firefly optimization based thresholding is applied for selecting the dynamic threshold values instead of fixed values. The firefly algorithm utilizes the objective function of the known optimization problem which depends on the light intensity and is used for fireflies to move towards brighter and the most attractive positions in order to acquire the optimal solution [16].

Initially, the population of fireflies referred as threshold values are initialized and assumed the light intensity variations and formulation of attractiveness. The attractiveness is computed based on the light intensity of fireflies which is determined by using the objective function. In the proposed system, the objective function is considered as Peak Signal-to-Noise Ratio (PSNR). The light intensity of the fireflies $I\left(f_{i}\right)$ is computed by using the objective function as follows:

$$
\text { Objective function, } f\left(x_{i}\right)=10 \log _{10} \frac{255^{2} . m \cdot n}{\sum_{i=1}^{m-1} \sum_{j=1}^{n-1}\left(x_{i j}-y_{i j}\right)^{2}}
$$

In equation (14), $x_{i j}$ is the pixel value for $(i, j)$ position of $\mathrm{X}$ frame, $y_{i j}$ is the pixel value for $(i, j)$ position of $\mathrm{Y}$ frame, and $m$ and $n$ are the size of windows.

$$
I\left(f_{i}\right)=\operatorname{Max}(P S N R), i=1,2, \ldots, n
$$

The attractiveness of fireflies is varied between firefly $i$ and $j$ based on their distance $r_{i j}$ and is measured as,

$$
\eta(r)=\eta_{0} \cdot e^{-\gamma r^{2}}
$$

In equation (16), $\eta_{0}$ represents the attractiveness at $r=0$. The light absorption coefficient $\gamma$ is computed as $\gamma=\frac{1}{\Gamma^{m}}$ where $\Gamma$ is called as the characteristic length scale in an optimization problem. The distance between two fireflies such as $i$ and $j$ at position $p_{i}$ and $p_{j}$ is computed based on the Cartesian distance.

$$
r_{i j}=\left\|p_{i}-p_{j}\right\|=\sqrt{\sum_{k=1}^{d}\left(x_{i, k}-x_{j, k}\right)^{2}}
$$

In equation (17), $x_{i, k}$ refers the $k^{\text {th }}$ component of the spatial coordinate $p_{i}$ of $i^{\text {th }}$ firefly. The movement of $i^{\text {th }}$ firefly to $j^{\text {th }}$ firefly which has more attractiveness is represented as follows:

$$
\begin{gathered}
p_{i}=p_{i}+\eta_{0} e^{-\gamma r_{i j}^{2}}\left(p_{j}-p_{i}\right)+\alpha \operatorname{sign}\left[\text { rand }-\frac{1}{2}\right] \oplus \text { Levy } \\
\text { Levy } \sim u=t^{-\lambda}, 1<\lambda \leq 3
\end{gathered}
$$

In equation (18), the first term represents the current position of $i^{\text {th }}$ firefly, the second term denotes the attractiveness of the firefly and the third term represents the randomization through the Levy flights where $\alpha$ is assumed as randomization parameter. The sign $\left[\right.$ rand $\left.-\frac{1}{2}\right]$, rand $\in[0,1]$ is used for providing the random sign 
or direction in which the random step length is obtained from the Levy distribution with infinite variance and mean.

Algorithm: Firefly Optimization based Thresholding

1. Initialize

2. Assign the objective function $f\left(x_{i}\right), i=1,2, \ldots, d$

3. Initialize the population of fireflies $f_{i}, i=1,2, \ldots, n$

4. For each firefly

5. Compute the light intensity $I\left(f_{i}\right)=\max f\left(x_{i}\right)$

6. Define light absorption coefficient $\gamma$

7. While $(t<$ MaxGeneration $)$

8. For $i=1: n$

9. For $j=1: i$

10. If $\left(I\left(f_{j}\right)>I\left(f_{i}\right)\right)$

11. Change attractiveness with distance $r$ through $e^{-\gamma r}$

12. Move $i^{\text {th }}$ firefly to $j^{\text {th }}$ firefly through Levy flights

13. Compute new solutions and update the light intensity

14. End If

15. End for $j$

16. End for $i$

17. Sort the fireflies and find the current best

18. End while

19. Obtain the optimal threshold value

20. End

Thus the proposed SVM and firefly based algorithms are used for image denoising based on the edge detection and segmentation effectively.

\section{EXPERIMENTAL RESULTS}

In this section, the performance of the proposed OAMNHA technique is evaluated for identifying its effectiveness compared with the existing MNHA approach without optimization. The target images along with different features are collected from the Laboratory for Image Video Engineering (LIVE) image database at the University of Texas at Austin [17]. The collected target images are considered as noise-free and noisy images where the noisy-images are consisting of noise-free images including with the Additive White Gaussian Noise (AWGN).

The different segmentation parameters are determined based on the target images. The computed parameters are varied based on the target images. Therefore, the optimal parameter values are selected by SVM in order to improve the edge segmentation performance. The smoothing process using bilateral filter is controlled by two standard deviations $\sigma_{d}$ and $\sigma_{r}$ and the window size. The size of window is selected as $5 \times 5$ and dynamic threshold values are used in canny edge detection process. The optimum threshold value is automatically selected by using firefly algorithm and also the optimum segmentation parameters are selected by SVM algorithm. The segmentation using the proposed approach results on Lena image are shown in fig. 1. In addition, fig. 2 illustrates the denoised images which are obtained by using the proposed OAMNHA method and existing MNHA method. Moreover, the comparison is made by using the performance metrics such as PSNR, Mean Absolute Error (MAE) and Structural Similarity Index (SSIM).

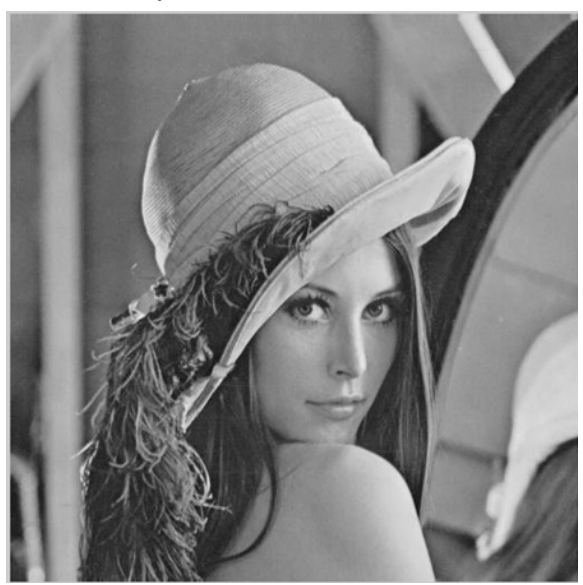

Fig.1 (a) Original Lena Image 


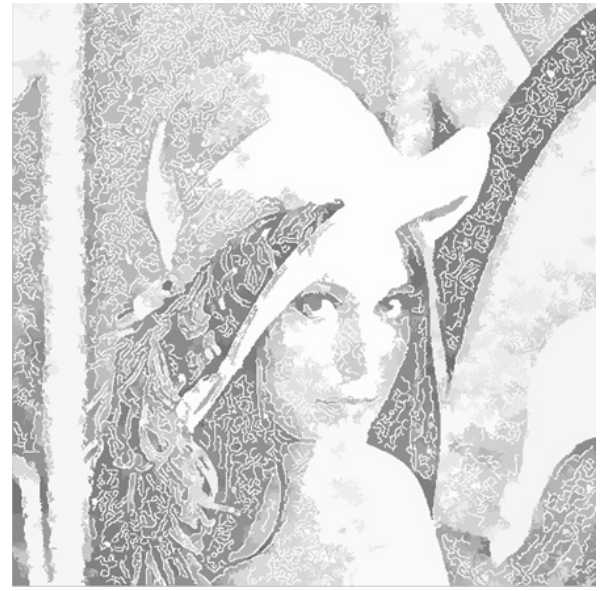

Fig.1 (b) Proposed Edge-preserving Segmentation Result of Lena Image

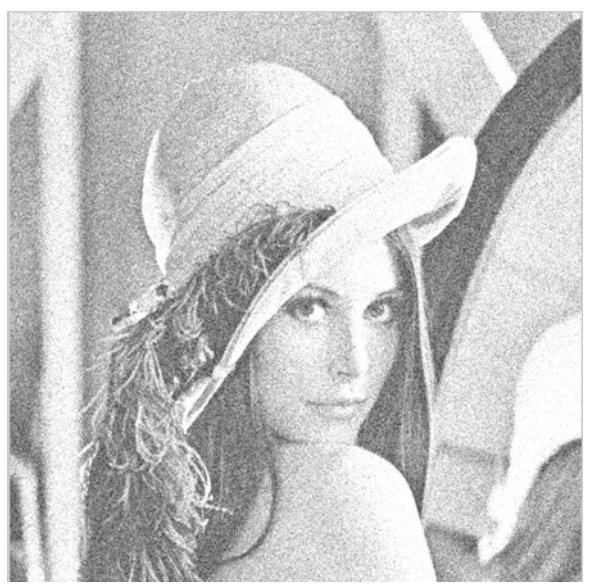

Fig.2 (a) Noisy Image $(\sigma=25)$

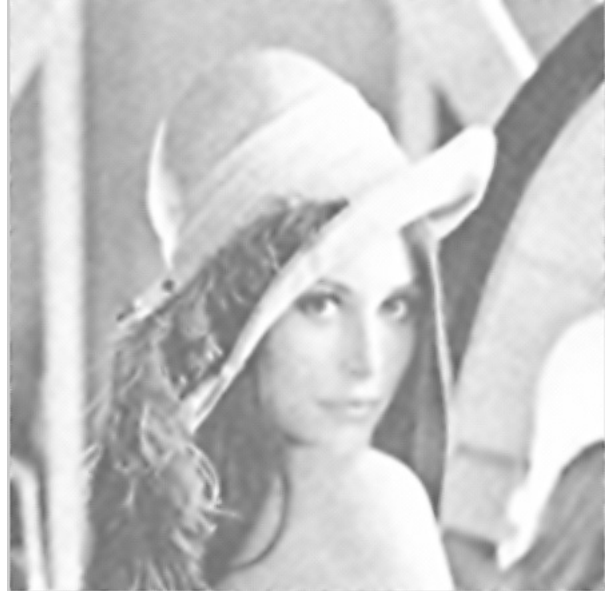

Fig.2 (b) Denoised Image using MNHA Method 


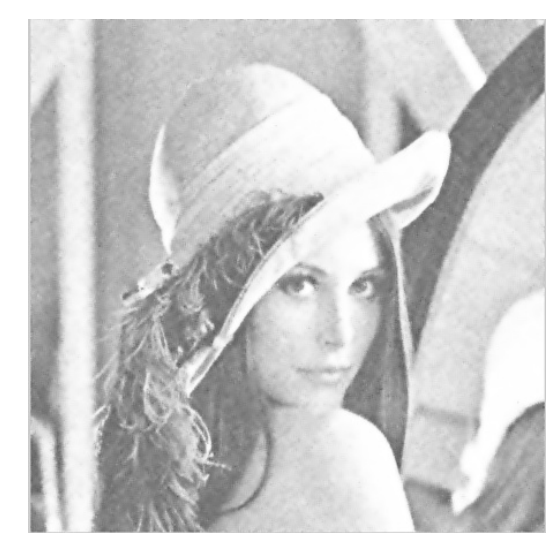

Fig.2 (c) Denoised Image using OAMNHA Method

\section{A. Peak Signal-to-Noise Ratio (PSNR)}

The PSNR is defined as the fraction of maximum possible signal power to the corrupting noise power which affects the fidelity of its representation. Generally, it is defined by using Mean Squared Error (MSE) and is computed as,

$$
\begin{gathered}
P S N R=10 \log _{10} \frac{255^{2}}{M S E} \\
\text { Where, } M S E=\frac{1}{m n} \sum_{i=1}^{m-1} \sum_{j=1}^{n-1}\left(x_{i j}-y_{i j}\right)^{2}
\end{gathered}
$$

The comparison of denoising PSNR which is made between proposed and existing approach is shown in table 1.

Table.1 Comparison of Denoising PSNR Results

\begin{tabular}{|c|c|c|c|c|c|c|}
\hline \multirow{2}{*}{$\begin{array}{c}\text { Noise } \\
\text { Intensity } \sigma\end{array}$} & \multicolumn{2}{|c|}{ Cameraman } & \multicolumn{2}{c|}{ Bikes } & \multicolumn{2}{c|}{ Ocean } \\
\cline { 2 - 7 } & MNHA & OAMNHA & MNHA & OAMNHA & MNHA & OAMNHA \\
\hline 5 & 38.50 & 40.25 & 39.35 & 41.58 & 40.81 & 42.63 \\
\hline 10 & 36.24 & 38.95 & 35.56 & 37.95 & 36.66 & 38.88 \\
\hline 15 & 33.88 & 35.48 & 32.88 & 35.62 & 34.12 & 36.95 \\
\hline 20 & 31.98 & 34.02 & 30.91 & 32.67 & 32.40 & 35.26 \\
\hline 25 & 30.53 & 32.68 & 29.45 & 32.22 & 31.12 & 33.41 \\
\hline 30 & 29.47 & 31.07 & 28.24 & 30.85 & 30.18 & 32.53 \\
\hline Noise & \multicolumn{2}{|c|}{ Statue } & \multicolumn{2}{|c|}{ Barbara } & \multicolumn{2}{|c|}{ Lena } \\
\cline { 2 - 8 } Intensity $\sigma$ & MNHA & OAMNHA & MNHA & OAMNHA & MNHA & OAMNHA \\
\hline 5 & 40.14 & 42.35 & 40.63 & 42.36 & 40.30 & 42.63 \\
\hline 10 & 36.84 & 38.65 & 36.93 & 38.44 & 37.34 & 39.86 \\
\hline 15 & 34.70 & 36.68 & 34.80 & 36.23 & 35.52 & 37.48 \\
\hline 20 & 33.05 & 35.49 & 33.22 & 35.41 & 34.26 & 36.09 \\
\hline 25 & 31.65 & 33.05 & 31.97 & 33.09 & 33.21 & 35.41 \\
\hline 30 & 30.53 & 32.77 & 30.90 & 32.31 & 32.40 & 34.22 \\
\hline Noise & \multicolumn{2}{|c|}{ Coinsfountain } & \multicolumn{2}{|c|}{ Lighthouse } & \multicolumn{2}{c|}{ Stream } \\
\cline { 2 - 8 } Intensity $\sigma$ & MNHA & OAMNHA & MNHA & OAMNHA & MNHA & OAMNHA \\
\hline 5 & 39.63 & 41.25 & 39.41 & 42.36 & 35.15 & 37.48 \\
\hline 10 & 35.57 & 37.48 & 35.86 & 37.55 & 32.75 & 34.65 \\
\hline 15 & 32.86 & 34.35 & 33.27 & 35.96 & 30.30 & 32.32 \\
\hline 20 & 31.00 & 33.03 & 31.72 & 33.06 & 28.43 & 30.93 \\
\hline 25 & 29.64 & 31.61 & 30.68 & 32.38 & 26.98 & 38.05 \\
\hline 30 & 28.62 & 30.94 & 29.93 & 31.88 & 25.93 & 37.40 \\
\hline
\end{tabular}


Table 1 shows that the proposed OAMNHA approach has better performance in terms of PSNR compared with the MNHA method.

\section{B. Mean Absolute Error (MAE)}

The MAE is defined as the quantity which is used for measuring the average magnitude of the error in the group of predictions and is computed as follows:

$$
M A E=\frac{1}{n} \sum_{i=1}^{n}\left|P_{i}-Y_{i}\right|=\frac{1}{n} \sum_{i=1}^{n}\left|e_{i}\right|
$$

In above equation, $P_{i}$ is the prediction value, $Y_{i}$ is the true value and $e_{i}$ is the absolute error. The comparison of denoising MAE for proposed approach and the existing method is shown in table 2.

Table.2 Comparison of Denoising MAE Results

\begin{tabular}{|c|c|c|c|c|c|c|}
\hline \multirow{2}{*}{$\begin{array}{c}\text { Noise } \\
\text { Intensity } \sigma\end{array}$} & \multicolumn{2}{|c|}{ Cameraman } & \multicolumn{2}{|c|}{ Bikes } & \multicolumn{2}{|c|}{ Ocean } \\
\hline & MNHA & OAMNHA & MNHA & OAMNHA & MNHA & OAMNHA \\
\hline 5 & 2.46 & 1.92 & 2.85 & 1.93 & 2.25 & 1.99 \\
\hline 10 & 3.43 & 2.80 & 4.46 & 3.84 & 3.34 & 3.15 \\
\hline 15 & 4.23 & 3.67 & 5.79 & 5.01 & 4.20 & 4.09 \\
\hline 20 & 5.15 & 4.55 & 7.00 & 6.24 & 4.95 & 4.44 \\
\hline 25 & 6.13 & 5.73 & 8.06 & 7.66 & 5.65 & 5.26 \\
\hline 30 & 6.98 & 5.88 & 9.09 & 8.76 & 6.24 & 5.77 \\
\hline \multirow{2}{*}{$\begin{array}{c}\text { Noise } \\
\text { Intensity } \sigma\end{array}$} & \multicolumn{2}{|c|}{ Statue } & \multicolumn{2}{|c|}{ Barbara } & \multicolumn{2}{|c|}{ Lena } \\
\hline & MNHA & OAMNHA & MNHA & OAMNHA & MNHA & OAMNHA \\
\hline 5 & 2.27 & 2.08 & 2.38 & 2.22 & 2.26 & 2.11 \\
\hline 10 & 3.27 & 3.11 & 3.54 & 3.36 & 3.12 & 2.94 \\
\hline 15 & 4.13 & 3.99 & 4.41 & 4.08 & 3.76 & 3.59 \\
\hline 20 & 5.00 & 4.69 & 5.20 & 4.79 & 4.33 & 4.03 \\
\hline 25 & 5.87 & 5.33 & 5.99 & 5.38 & 4.87 & 4.49 \\
\hline 30 & 6.70 & 5.89 & 6.73 & 6.04 & 5.34 & 4.92 \\
\hline \multirow{2}{*}{$\begin{array}{c}\text { Noise } \\
\text { Intensity } \sigma\end{array}$} & \multicolumn{2}{|c|}{ Coinsfountain } & \multicolumn{2}{|c|}{ Lighthouse } & \multicolumn{2}{|c|}{ Stream } \\
\hline & MNHA & OAMNHA & MNHA & OAMNHA & MNHA & OAMNHA \\
\hline 5 & 2.78 & 2.65 & 2.57 & 2.36 & 4.07 & 3.38 \\
\hline 10 & 4.25 & 4.08 & 3.69 & 3.42 & 6.02 & 5.92 \\
\hline 15 & 5.45 & 5.31 & 4.62 & 4.38 & 7.61 & 7.44 \\
\hline 20 & 6.51 & 6.35 & 5.39 & 5.10 & 9.06 & 8.85 \\
\hline 25 & 7.47 & 7.19 & 6.03 & 5.73 & 10.32 & 10.11 \\
\hline 30 & 8.40 & 8.02 & 6.57 & 6.15 & 11.39 & 11.02 \\
\hline
\end{tabular}

Table 2 shows that the proposed OAMNHA approach has better performance in terms of MAE compared with the MNHA method.

\section{Structural Similarity Index (SSIM)}

It is defined as the similarity value between any two images $(x, y)$ and is computed as follows:

$$
\operatorname{SSIM}(x, y)=\frac{\left(2 \mu_{x} \mu_{y}+c_{1}\right)\left(2 \sigma_{x y}+c_{2}\right)}{\left(\mu_{x}^{2}+\mu_{y}^{2}+c_{1}\right)\left(\sigma_{x}^{2}+\sigma_{y}^{2}+c_{2}\right)}
$$

In the above equation, the averages of $x$ and $y$ are represented as $\mu_{x}$ and $\mu_{y}$. Also, the variances of $x$ and $y$ are represented as $\sigma_{x}^{2}$ and $\sigma_{y}^{2} x, y$ respectively, $c_{1}$ and $c_{2}$ are constants. In addition, the covariance of $x$ and $y$ is denoted as $\sigma_{x y}$. The comparison of denoising SSIM for proposed approach and the existing approach is shown in table 3 . 
Table.3 Comparison of Denoising SSIM Results

\begin{tabular}{|c|c|c|c|c|c|c|}
\hline \multirow{2}{*}{$\begin{array}{c}\text { Noise } \\
\text { Intensity } \sigma\end{array}$} & \multicolumn{2}{|c|}{ Cameraman } & \multicolumn{2}{c|}{ Bikes } & \multicolumn{2}{c|}{ Ocean } \\
\cline { 2 - 7 } & MNHA & OAMNHA & MNHA & OAMNHA & MNHA & OAMNHA \\
\hline 5 & 0.965 & 0.980 & 0.984 & 0.988 & 0.971 & 0.981 \\
\hline 10 & 0.946 & 0.962 & 0.958 & 0.975 & 0.930 & 0.953 \\
\hline 15 & 0.917 & 0.938 & 0.923 & 0.944 & 0.883 & 0.896 \\
\hline 20 & 0.881 & 0.893 & 0.885 & 0.909 & 0.838 & 0.860 \\
\hline 25 & 0.838 & 0.871 & 0.847 & 0.861 & 0.797 & 0.822 \\
\hline 30 & 0.801 & 0.860 & 0.809 & 0.820 & 0.763 & 0.801 \\
\hline Noise & \multicolumn{2}{|c|}{ Statue } & \multicolumn{2}{|c|}{ Barbara } & \multicolumn{2}{|c|}{ Lena } \\
\cline { 2 - 8 } Intensity $\sigma$ & MNHA & OAMNHA & MNHA & OAMNHA & MNHA & OAMNHA \\
\hline 5 & 0.966 & 0.974 & 0.973 & 0.980 & 0.952 & 0.966 \\
\hline 10 & 0.932 & 0.948 & 0.950 & 0.962 & 0.923 & 0.938 \\
\hline 15 & 0.899 & 0.913 & 0.933 & 0.948 & 0.901 & 0.922 \\
\hline 20 & 0.864 & 0.882 & 0.915 & 0.935 & 0.883 & 0.903 \\
\hline 25 & 0.823 & 0.860 & 0.895 & 0.911 & 0.866 & 0.890 \\
\hline 30 & 0.784 & 0.841 & 0.873 & 0.896 & 0.853 & 0.879 \\
\hline Noise & \multicolumn{2}{|c|}{ Coinsfountain } & \multicolumn{2}{|c|}{ Lighthouse } & & Stream \\
\cline { 2 - 7 } Intensity $\sigma$ & MNHA & OAMNHA & MNHA & OAMNHA & MNHA & OAMNHA \\
\hline 5 & 0.976 & 0.981 & 0.964 & 0.972 & 0.976 & 0.987 \\
\hline 10 & 0.939 & 0.952 & 0.926 & 0.941 & 0.941 & 0.963 \\
\hline 15 & 0.892 & 0.916 & 0.873 & 0.898 & 0.876 & 0.899 \\
\hline 20 & 0.844 & 0.869 & 0.830 & 0.847 & 0.802 & 0.824 \\
\hline 25 & 0.803 & 0.820 & 0.801 & 0.825 & 0.732 & 0.758 \\
\hline 30 & 0.769 & 0.788 & 0.781 & 0.806 & 0.677 & 0.695 \\
\hline
\end{tabular}

Table 3 shows that the proposed OAMNHA approach has better performance in terms of SSIM compared with the MNHA method.

\section{CONCLUSION}

The image denoising is the most challenging process in image processing techniques. Hence, in this paper, an efficient image denoising technique by using the high-frequency resolution analysis is proposed. In the proposed approach, the novel technique called Mask NHA is proposed including with the edge preservation and segmentation approach. The edge preserving method is performed based on the canny edge detection and mean shift-based segmentation by utilizing the different segmentation parameters. In addition, these segmentation parameters are optimized by the proposed machine learning SVM algorithm for precise segmentation. Furthermore, the edge detection in denoised images is achieved based on the thresholding approach. Therefore, in the proposed approach, the optimal threshold values are selected by using the firefly optimization algorithm. Finally, the experimental results of the proposed approach are compared with the state-of-arts methods in order to distinguish the effectiveness of the proposed algorithm. It proves that the proposed OAMNHA approach has higher PSNR, SSIM and lower MAE values compared to the MNHA based image denoising approach.

\section{REFERENCES}

[1] Rajni, R., \& Anutam, A. (2014). Image denoising techniques-An overview. International Journal of Computer Applications, 86(16), 13-17.

[2] Pathak, M., \& Singh, S. (2014). Comparative analysis of image denoising techniques. International Journal of Computer Science \& Engineering Technology, 1(5), 160-167.

[3] Singh, R., Singh, P., \& Parveen, F. (2015). Brief review on image denoising techniques. International Journal of Science, Technology \& Management, 4(1), 336-344.

[4] Hosotani, F., Inuzuka, Y., Hasegawa, M., Hirobayashi, S., \& Misawa, T. (2015). Image Denoising With Edge-Preserving and Segmentation Based on Mask NHA. IEEE Transactions on Image Processing, 24(12), 6025-6033.

[5] Yoshizawa, T., Hirobayashi, S., \& Misawa, T. (2011). Noise reduction for periodic signals using high-resolution frequency analysis. EURASIP Journal on Audio, Speech, and Music Processing, 2011(1), 5.

[6] Xiao, Y., Zeng, T., Yu, J., \& Ng, M. K. (2011). Restoration of images corrupted by mixed Gaussian-impulse noise via 1 1-l 0 minimization. Pattern Recognition, 44(8), 1708-1720.

[7] Hasegawa, M., Kako, T., Hirobayashi, S., Misawa, T., Yoshizawa, T., \& Inazumi, Y. (2013). Image inpainting on the basis of spectral structure from 2-D nonharmonic analysis. IEEE Transactions on Image Processing, 22(8), 3008-3017.

[8] Yang, S., Zhao, L., Wang, M., Zhang, Y., \& Jiao, L. (2013). Dictionary learning and similarity regularization based image noise reduction. Journal of Visual Communication and Image Representation, 24(2), 181-186. 
[9] Zhang, J., \& Hirakawa, K. (2017). Improved Denoising via Poisson Mixture Modeling of Image Sensor Noise. IEEE Transactions on Image Processing, 26(4), 1565-1578.

[10] Xiong, R., Liu, H., Zhang, X., Zhang, J., Ma, S., Wu, F., \& Gao, W. (2016). Image Denoising via Bandwise Adaptive Modeling and Regularization Exploiting Nonlocal Similarity. IEEE Transactions on Image Processing, 25(12), 5793-5805.

[11] Ma, H., \& Nie, Y. (2016). An edge fusion scheme for image denoising based on anisotropic diffusion models. Journal of Visual Communication and Image Representation, 40, 406-417.

[12] Fan, F., Ma, Y., Li, C., Mei, X., Huang, J., \& Ma, J. (2017). Hyperspectral image denoising with superpixel segmentation and lowrank representation. Information Sciences, 397, 48-68.

[13] Vandana, M., \& Srinivas, R. (2011). An optimized algorithm for ringing region detection in compressed images. International Journal of Modern Engineering Research (IJMER), 1(2), 261-269.

[14] Ghassabeh, Y. A. (2015). A sufficient condition for the convergence of the mean shift algorithm with Gaussian kernel. Journal of Multivariate Analysis, 135, 1-10.

[15] Awad, W. A., \& Elseuofi, S. M. (2011). Machine learning methods for spam e-mail classification. International Journal of Computer Science \& information Technology (IJCSIT), 3(1), 173-184.

[16] Yang, X. S. (2010). Firefly algorithm, Levy flights and global optimization. In Research and development in intelligent systems XXVI (pp. 209-218). Springer London.

[17] http://live.ece.utexas.edu/research/quality/subjective.html 